\title{
Age and Treatment Related Local Hippocampal Changes in Schizophrenia Explained by a Novel Shape Analysis Method
}

\author{
Guido Gerig ${ }^{1,2}$, Keith E. Muller ${ }^{3}$, Emily O. Kistner ${ }^{3}$, Yueh-Yun Chi ${ }^{3}$, \\ Miranda Chakos ${ }^{2}$, Martin Styner ${ }^{1}$, and Jeffrey A. Lieberman ${ }^{2}$ \\ 1 Departments of Computer Science \\ 2 Psychiatry \\ gerig@cs.unc.edu ${ }^{\dagger}$ \\ 3 Biostatistics \\ University of North Carolina, Chapel Hill, NC 27599, USA
}

\begin{abstract}
Volume reduction of the hippocampus observed with MRI is one of the most consistently described structural abnormalities in patients with schizophrenia. However, the timing, the association with treatment, and an intuitive explanation of morphologic changes in terms of shape differences are not known. This study analyzed subtle changes of the hippocampal structure in schizophrenics as compared to matched controls. The effect of age, duration of illness and treatment effects to local shape changes was studied with a new shape representation technique and an exploratory statistical analysis. Shape representation was based on a sampled medial model (M-rep) describing each shape by an 8 by 3 mesh with local position and width at each node. This model allows an independent analysis of shape deformation and of local atrophy. The exploratory statistical model was a repeated measures ANOVA, cast as a general linear multivariate model. The new procedure overcomes the problem of testing a very large number of correlated observations, which is a significant limitation of many other shape discrimination schemes. As a novel contribution not shown before, it models shape in combination with patient variables to answer important clinical questions in regard to drug response and longitudinal change in order to assess developmental and degenerative processes. The exploratory nature of the analysis means that our results must be replicated in a hypothesis driven independent patient study in order to provide full confidence in the conclusions.
\end{abstract}

\section{Introduction}

Schizophrenia is often subject to conflicting hypotheses about the cause and temporal evolution of the neuropathologic features of the disorder and its relationship to treatment. Volume reduction of the hippocampus is one of the most

\footnotetext{
$\dagger$ This work was supported by the NCI grant P01 CA47982. The MRI hippocampal study of schizophrenics was funded in part by the Stanley Foundation, the Foundation of Hope and the UNC-MHNCRC (MH33127). A color paper version is available at: www.cs.unc.edu/ gerig.
} 
consistently described structural abnormalities in patients with schizophrenia. However, the cause and timing of this pathomorphologic feature of the illness is not known. Csernansky et al. 12] suggests that a full characterization of neuroanatomical abnormalities will increase our understanding of etiology, pathogenesis, and pathophysiology of schizophrenia. Results show that the analysis of hippocampal shape discriminates schizophrenia and control subjects with greater power than volumetry [342]. Most recently, Shenton et al. [5] found that hippocampal volume and shape provided much better discrimination than each of these variables alone. All these studies suggest a clear need for studying shape rather than gross volume and for providing quantitative measures that are not only statistically significant but also neuroanatomically meaningful and intuitive.

The effects of drug treatment have been an issue that has complicated the interpretation of studies of neuropathology and brain morphology in schizophrenia. Most significant for patient outcome, however, is the question if a certain drug treatment might prevent or delay loss of structure such as atrophy of hippocampus. A structure description based on medial object models, as used here, captures global and local object shape by the shape of its set of medial manifolds and by local width (radius) measurements. Such a description might have advantages over conventional surface-based or high-dimensional voxel deformation based descriptions 67. Medial manifold descriptions potentially provide us with natural and intuitive measurements such as global length, width as well as local thickness and bending 8 .

In the hippocampal shape analysis study presented here, we not only ask the scientific question if schizophrenics and controls differ in hippocampal shape but try to link quantitative morphologic measurements to treatment drug type and to longitudinal change with age or duration of illness. In this novel concept, both patient variables and shape parameters are becoming part of the statistical model.

\section{Methods}

\subsection{Data, Subjects, Segmentation, Shape Model}

Subjects. The patient study is cross-sectional in design [9] and assesses potential differences in the hippocampus structure between patients in the first few years of illness versus patients who have been chronically ill. Early illness (age 16-30, $\mathrm{N}=34$ ) and chronic (age 31-60, $\mathrm{N}=22$ ) patients have been matched to a young and an older control group $(\mathrm{N}=26)$. Patients were characterized with regards to duration of illness and illness severity utilizing PANSS assessments. All patients and comparison subjects were right-handed male. At the time of the scan, 22 patients were on typical antipsychotic medication (haloperidol), and 35 were on atypical antipsychotic medications (olanzapine, risperidone).

Imaging and Segmentation. Patients were scanned on a GE Sigma Advantage MR system operating at 1.5 Tesla. The series used for this study was acquired as a 3D IR Prepped Fast SPGR, FOV=24cm; 256 256, 124 slices with $1.5 \mathrm{~mm}$ thickness. Analysis of the hippocampus was performed using the software package for 

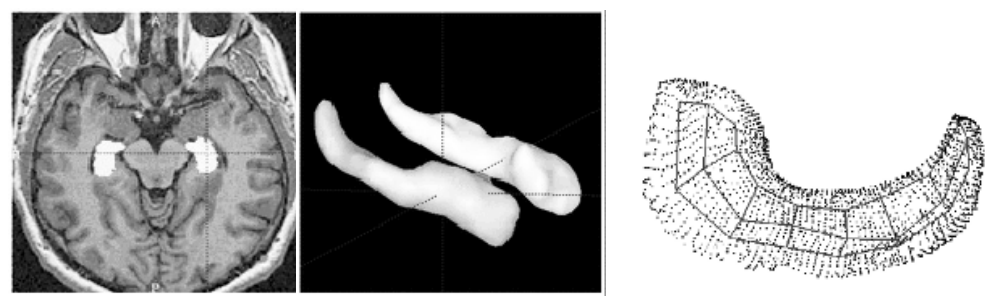

Fig. 1. Anatomical location of left and right hippocampal structures (left), 3-D rendering of the structures (middle) and the M-rep shape representation model used in this analysis (right).

three-dimensional segmentation IRIS (free download at midag.cs.unc.edu). Hippocampal segmentation was based on a well-documented protocol and included the hippocampus proper, the subiculum, the fimbria, and subsplenial gyrus. All measurements were completed by a single rater (S.A.S.) whose intrarater reliability was 0.86 for the left hippocampus and 0.88 for the right hippocampus.

Shape Representation. We used a shape analysis pipeline to parametrize the surface of each object, to provide an area preserving mapping to a sphere with uniform sampling, and to calculate the minimal M-rep sampling necessary given an error criterion for object overlap and mean absolute boundary distance. Shapes were aligned using Procrustes fit at the boundary but we did not include normalization for size since the analysis as used herein naturally separates scale. These procedures have been described elsewhere [6] and will not be discussed here. The final M-rep representation for the population of 79 left and right hippocampal shape was determined as a $3 x 8$ grid of mesh points (see Fig. 1 A simplified M-rep representation with $\{x, y, z\}$ and radius for each of the 24 mesh nodes was used.

\subsection{Statistical Model}

Data Structure. Data were reduced to two outcome measures: Euclidean distance in $\mathrm{mm}$ from some common origin across all hippocampi was the first outcome of interest. This metric reduces the $\{x, y, z\}$-tuple to a single distance and avoids to model interrelationships among $x, y$ and $z$ and their variances. Radius in $\mathrm{mm}$ as local thickness (24 locations per hippocampus) was the second outcome of interest. Each outcome was analyzed completely separately from the other. For each analysis, the within subject effects included Side (of brain), Row, and Column (in M-rep structure). Each of the two Y matrices are $79 \times 48$, with 79 subjects and 24 M-rep points per side of the hippocampus (Left and Right). Predictors for within subject effects in the model model were: Side, Row, Column, Side x Row, Side x Column, Row x Column, and Side x Row x Column $(10+21+14$ parameters). Predictors for between subject effects in the model model were: Age (years), Drug_Type (none, typical, or atypical), Drug_Type x Age (2 parameters) interaction, and Drug_Type x Duration (8 parameters). 
Model Reduction. Analysis began with a residual analysis conducted on the full model on both outcomes of interest. The approach followed recommendations as found in [10. The model reduction follows a planned, fixed sequence of tests, always from a larger to smaller model (1112). The exploratory analysis requires us to report all steps of our process to avoid misleading interpretation of the results. The multiple steps for selecting the optimal models are shown in the Appendix 4 with the example of the deformation model.

\section{$3 \quad$ Results}

The results of this exploratory analysis scheme must be interpreted with caution due to the large number of data-driven features. A novel contribution not shown before in clinical shape analysis studies, we separately tested for differences in local width and local deformation, and modelled shape in combination with patient variables. It is important to emphasize that both measures, the Euclidean Distance and the Radius Distance, are differential measures. Shapes are normalized by average distance from origin and average radius. The measures thus represent pure shape deformation and pure radius deformation measures after normalization. This type of shape analysis is different from an analysis of objects of different size and asking for the location of major size differences, although this question could be explored using a similar analysis scheme.

Global size differences. Our shape modelling scheme allows us to infer global volume indirectly via M-rep radius using the statistical analysis framework presented here. The integrated radius measure serves as an indicator of global size. The global hippocampal volume, obtained by averaging the m-rep radius feature (Log2(Radius)) over Side, Row and Column, was put into the exploratory analysis scheme (44). The final model included Age, Drug Type(Atyp, Typ, Cntl), $\operatorname{Drug}($ yes, no)*Age and Drug(yes, no)*Duration as predictors. The volume difference between Typical and Control, and between Atypical and Control are not significant, but the hippocampal volumes of the Atypical and Typical groups show a significant difference $(\mathrm{p}<0.0234)$.

Euclidean Distance Results. The final model for distance included Age, Drug (yes, no), and Age x Drug as predictors, with distance averaged across side of the hippocampus (left/right) as the outcome of interest. The difference in hippocampus shape between patients and controls as measured by M-rep distance is represented by Fig. 2. Figures left to right represent the difference at age 20, 30 and 40, respectively. The difference in hippocampus shape deformation between patients and controls is located mostly in the tails of the hippocampus, and becomes more pronounced over time. Figure 4 left represents the change in hippocampus shape over ten years for a control and demonstrates a very small longitudinal deformation change.

Radius Distance Results. The final model for M-rep radius included Age, DrugType (none, typical, atypical), the interaction of Age x Drug (yes, no), and the interaction of Duration x Drug (yes, no) as predictors, with the difference of 


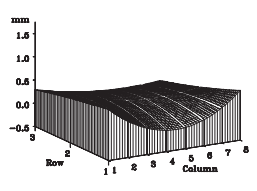

Age 20

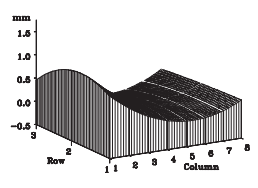

Age 30

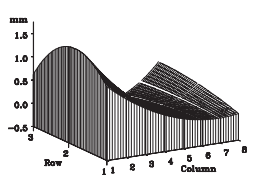

Age 40

Fig. 2. Patient - Control Euclidean distance differences (shape deformation, bending) at age 20, 30 and 40, left to right. The $\mathrm{x}$ - and $\mathrm{y}$-axis represent column and row of the 3x8 mesh M-rep representation. Height indicates the deformation difference between patient and controls in $\mathrm{mm}$. The graphs characterize time slices of the continuous age range. The differences between patients and controls increase over time and are located mostly in the tail and to a lesser extent in the head of the hippocampus.

distance between side (left - right) of the hippocampus as the outcome of interest. The following figures (Fig[3) illustrate a reduction in difference in M-rep distance between patients and controls over time. Please note that the vertical axis is a difference of differences, namely Patient(Left-Right) - Control(Left-Right). The difference in hippocampus shape between the typical drug group and controls and atypical drug group and controls as measured by M-rep radius is represented by Figures 3 left and right column, top to bottom, one figure for each decade (age 20 , age 30 , and age 40 ).

Age Typical Drug - Control

Age 40

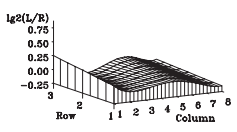

Age 30

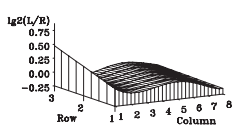

Age 20
Atypical Drug - Control
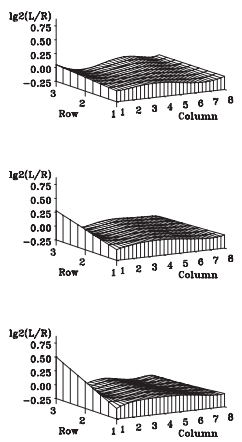

Fig. 3. Drug - Type versus Control group differences of left to right radius difference $\log 2(L / R)$, which can be interpreted as $\mathrm{L}$ to $\mathrm{R}$ local width asymmetry. Left Column: Typical Drug - Control radius asymmetry difference at age 20, 30 and 40, top to bottom. Right Column: Same for Atypical Drug - Control radius asymmetry. The x- and $\mathrm{y}$-axis represent column and row of the $3 \mathrm{x} 8$ mesh M-rep representation. Height indicates asymmetry of the radius difference (difference of differences) between patient and controls in $\mathrm{mm}, \log _{2}$ scaled. The graphs characterize time slices of the continuous age range and show decreasing asymmetry of local width with age, particularly in the tail and head regions of the hippocampi. 
Comparison: Longitudinal shape change of Controls. Figure 4 illustrates the change in hippocampus shape and local width over ten years for controls using the final models for Deformation (left) and Radius (right). The vertical axis represents deformation in $\mathrm{mm}$ and a difference of differences, namely Control (Left-Right) - Control(Left-Right), respectively. Both figures demonstrate the very small changes for controls over the 10 years interval.
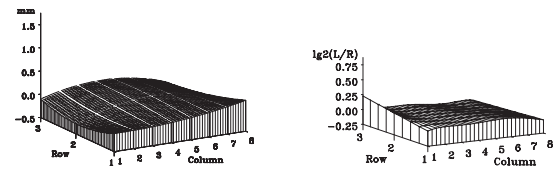

Fig. 4. Deformation and width asymmetry changes due to aging within controls for 10 years. Left: Distance model representing shape deformation, scale in mm. Right: Radius model representing left/right asymmetry of local radius change (local width), $\log _{2}$-scaled in $\mathrm{mm}$.

Table 1. Summary of final statistical models, tests and significance values.

\begin{tabular}{|c|c|c|c|}
\hline Type of Shape Change & Between subject model & Test & Significance \\
\hline $\begin{array}{l}\text { Global Volume } \\
\text { Integrated Radius Differ- } \\
\text { ence }\end{array}$ & $\begin{array}{l}\text { Age }+\operatorname{Drug}(\text { Typ,Atyp,None) } \\
+\quad \text { Age } x \operatorname{Drug}(y / n) \\
\text { Duration } x \operatorname{Drug}(y / n)\end{array}$ & Drug & $\mathrm{p}=0.0234 * *$ \\
\hline $\begin{array}{l}\text { Shape Deformation } \\
\text { Distance difference }\end{array}$ & $\begin{array}{l}\text { Age }+ \text { Drug }(y / n)+\text { Age } x \text { Drug } \\
(y / n)\end{array}$ & Row $x$ Col x Drug $x$ Age & $\mathrm{p}=0.0091 * *$ \\
\hline $\begin{array}{l}\text { Local Width Change } \\
\text { Radius difference }\end{array}$ & $\begin{array}{l}\text { Age }+ \text { Drug(Typ,Atyp,None) }+ \\
\operatorname{Drug}(y / n)+\text { Age } x \text { Drug }(y / n)\end{array}$ & Row x Col x Drug x Age & $\begin{array}{l}p=0.1213 \text { (Left) } \\
p=0.0998 \text { (Right) }\end{array}$ \\
\hline $\begin{array}{l}\text { Width Asymmety } \\
\text { Change } \\
\text { Difference of } L / R \text { radius } \\
\text { asymmetry }\end{array}$ & $\begin{array}{l}\text { Age }+\operatorname{Drug}(\text { Typ, Atyp,None })+ \\
\text { Age } x \operatorname{Drug}(y / n)\end{array}$ & $\begin{array}{l}\text { Side } \mathrm{x} \text { Row } \mathrm{x} \text { Col } \mathrm{x} \text { Drug } \mathrm{x} \text { Dur. } \\
\text { Side } \mathrm{x} \text { Row } \mathrm{x} \text { Col } \mathrm{x} \text { Drug } \mathrm{x} \text { Age }\end{array}$ & $\begin{array}{l}\mathrm{p}=0.0077 * * \\
\mathrm{p}=0.0421 * *\end{array}$ \\
\hline
\end{tabular}

\section{Discussion}

We present a statistical analysis of anatomical shape integrated into a statistical framework that includes patient variables. Treating shape, age, and drug treatment as variables in a statistical model is new and has not been shown before. This analysis will potentially lead to answers in regard to progression of shape change with different drug treatments or with neurodegeneration, both highly relevant for research in the field of psychiatry. The global volume differences between Typical and Control, and also between Atypical and Control, are not significant. The hippocampal volumes of the Atypical and Typical groups, however, show a significant difference. The Typical group shows smaller hippocampal volume than the Atypical group. The Deformation measure shows a 
significant shape change between controls and schizophrenics, but not for subgroups treated with different drugs and not for side (left,right). This deformation confirms earlier shape analysis of the same shapes using spherical harmonics and cpoint distribution models [8]. The tail of the hippocampus of schizophrenics is flattened in comparison to the stronger bending of the tail in controls. A smaller effect is found in the hippocampal head at the hippocampal-amygdala transition region, confirming recent shape findings by Csernansky [2]. The Radius analysis shows that locations at the head and tail of the object, not the center, present differences. Differences between patients and controls in hippocampal radius asymmetry decrease over time. This reduction in group difference (of $\mathrm{L} / \mathrm{R}$ difference) seems more pronounced in the Atypical group. The Atypical treated patients start (at an early age) less far from the Controls than do Typical treated, which might be interpreted as treatment effect or a clinical selection bias.

The current approach reduces shape deformation at mesh nodes to simple distances. This data reduction approach provides only limited information about object deformations and cannot completely differentiate between bending and elongation, for example, as both changes would have a similar effect on this variable.

Following this exploratory study, we will continue with an confirmatory analysis in an independent schizophrenia study with fixed hypothesis prior to data collection.

\section{Appendix}

The following paragraph describes details of the steps of our exploratory analysis scheme. Due to space limitation, we only present the Euclidean Distance Analysis (Long paper version: http://www.cs.unc.edu/rgerig). Euclidean Distance Analysis: The residual analysis of distance showed no serious violation of the assumptions. The Univariate Approach to Repeated Measures (UNIREP) was considered appropriate based on the estimate of epsilon (0.56). This value plus the pattern of p-values led us to focus on the Geisser-Greenhouse tests for distance.

1. Tests of interaction of Drug - Type x Duration with all combinations of Side, Row, and Column. None of the p-values were less than .05.

2. Reduction of between-subject model to one with a common slope for DrugType $\mathrm{x}$ Duration (equivalent to a model with the main effect of Duration because all controls have zero Duration). Tests also gave p-values greater than .05. Therefore Drug - Type x Duration was dropped from the model.

3. Reduction of between subject model including Age, Drug - Type, and the interaction of Age x Drug - Type. Conduction of tests of interaction of Drug-Type x Age with all combinations of Side, Row, and Column. Row x Col x Drug - Type x Age gave a p-value of 0.026, estimate of epsilon: 0.52.

4. Average over Side which reduces the Y matrix to a $79 \times 24$ matrix, with each column corresponding to the (leftpoint + rightpoint) $/ 2$. Testing equality of the Drug - Type x Age slopes. The test gave a p-value greater than .05. 
5. Reduction to a model with a common slope for Drug-Type x Age (equivalent to the simple interaction of Drug (yes, no) and Age). The test of Row x Col x Drug x Age gave a smaller p-value in this model ( $\mathrm{p}$-value $=0.0113$ ). A test of a equality of intercepts (difference in Drug-Types, typical versus atypical) was found to give $\mathrm{p}>.05$.

6. Final reduction of the between subject model, including Age, Drug (yes, no), and Age x Drug as predictors. The test of Row x Col x Drug x Age in the final model led to $\mathrm{p}=0.0097$ (Geisser-Greenhouse test).

7. The Row x Column shape was judged to be a quadratic (Row) by quadratic (Column) predicted surface (step-down interaction trend test).

\section{References}

1. Csernansky, J., Joshi, S., Wang, L., Haller, J., Gado, M., Miller, J., Grenander, U., Miller, M.: Hippocampal morphometry in schizophrenia via high dimensional brain mapping. Proc. Natl. Acad. Sci. USA 95 (1998) 11406-11411

2. Csernansky, J., Wang, L., Jones, D., Rastogi-Cruz, D., Posener, J., Heydebrand, G., Miller, J., Miller, M.: Hippocampal deformities in schizophrenia characterized by high dimensional brain mapping. Am J Psychiatry 159 (2002) 1-7

3. Haller, J.W., Christensen, G.E., Joshi, S., , Newcomer, J.W., Miller, M.I., Csernansky, J.C., Vannier, M.W.: Hippocampal mr imaging morphometry by means of general pattern matching. Radiology 199 (1996) 787-791

4. Wang, L., Joshi, S.C., Miller, M.I., Csernansky, J.G.: Statistical analysis of hippocampal asymmetry in schizophrenia. in press, NeuroImage (2000)

5. Shenton, M., Gerig, G., McCarley, R., Szekely, G., Kikinis, R.: Amygdalahippocampus shape differences in schizophrenia: The application of 3d shape models to volumetric mr data. Psychiatry Research Neuroimaging 115 (2002) 15-35

6. Styner, M., Gerig, G.: Three-dimensional medial shape representation incorporating object variability. In: Proc. of Computer Vision and Pattern Recognition CVPR 2001, IEEE Computer Society (2001) 651-656

7. Pizer, S., Joshi, S., Fletcher, T., Styner, M., Trecton, G., Chen, J.: Object models in multiscale intrinsic coordinates via m-reps. In: Med. Im. Comp. and ComputerAssist. Interv. MICCAI 2002. Volume 2489 of LNCS. (2002) 862-871

8. Gerig, G., Styner, M., Shenton, M., Lieberman, J.: Shape versus size: Improved understanding of the morphology of brain structures. In: MICCAI 2002. Volume 2208 of LNCS., Springer (2001) 24-32

9. Chakos, M., Schobel, S., Gerig, G., Gu, H., Charles, C., Lieberman, J.: Clinical correlates of hippocampal volume in schizophrenia as assessed by $3 \mathrm{~d}$ manual segmentation. submitted to Archives of General Psychiatry (2002)

10. KE, M., BA., F.: A Unified Approach to Regression and ANOVA: Theory and Applications Using SAS Software. Cary, NC: SAS Institute. (2002)

11. Muller, K., LaVange, L., Ramey, S., , Ramey, C.: Power calculations for general linear multivariate models including repeated measures applications. Journal of the American Statistical Association 87 (1992) 1209-1226

12. Muller, K., Pasour, V.: Bias in linear model power and sample size calculation due to estimating variance. Communcations in Statistics: Theory and Methods 26 (1997) 839-851 